\title{
Synthesization of Thermally Induced Errors in Coordinate Measuring Machines
}

B. Di Giacomo

and F. T. Paziani

Escola de Engenharia de São Carlos Av. Trabalhador Sãocarlense N.400, Centro 13566-590 São Carlos, SP. Brazil arvaldes@sc.usp.br bgiacomo@sc.usp.br fpaziani@sc.usp.br
This work presents the equations for the components of the volumetric error of a Coordinate Measuring Machine (CMM) considering thermal influences. These equations were applied to a moving bridge CMM and combines homogeneous transformations, regression techniques and least squares algorithm. The magnitudes of both the geometric errors and its thermally induced variations were collected by means of a Laser interferometric system, a mechanical square and an electronic level. Simultaneously, temperature values were monitored using T-type Copper-Constantan thermocouples. From the proposed model, components of the volumetric errors were synthesized. Results are discussed and compared to the ones obtained from measurement sets of a ring gauge. It was verified that the model presented an excellent ability to predict volumetric error of the CMM. Errors of about 10 um in magnitude were reduced by at least $75 \%$, while for errors greater than $10 \mu \mathrm{m}$, the efficiency of the model was $90 \%$.

Keywords: Thermal drift, thermally induced errors, thermal states, spatial thermal gradients and hot spots

\section{Introduction}

The manufacturing of products within smaller tolerances and in larger quantities has impelled the necessity of developing faster, more accurate, flexible and reliable quality control approaches. To accomplish these objectives, Coordinate Measuring Machines can be employed. Despite their known advantages, the working performance of CMMs is limited by several factors that take action simultaneously, combining themselves in a complex manner over the entire working volume, producing volumetric errors.

Geometric errors compose the most representative fraction of the volumetric error (Bosch, 1995). These errors derive from the geometric deviations of different components of the CMM and emerge during the motion of the coordinate axes due to components interaction, affecting on the relative positioning between probe and part, altering the measurement result. ${ }^{1}$

In order to study geometric errors, the CMM moving elements are assumed as rigid bodies. The position of a rigid body in space can be defined by six degrees of freedom. Since each degree of freedom can be associated to an error, six geometric errors are associated to each preferential axis of the CMM, specifically, one position error, two straightness errors and three rotation errors (pitch, yaw and roll), summing up a total number of 18 geometric errors. Three more errors must be added due to the impossibility of arranging three perfectly orthogonal axes, namely orthogonal errors, which depend on the relation between components. Therefore, a full amount of 21 errors can be determined from three axis Cartesian CMMs.

At the temperature of $20^{\circ} \mathrm{C}$, geometric errors can be considered constant, once they vary very slowly with time. However, if the temperature is different from $20^{\circ} \mathrm{C}$, these errors can change in magnitude and behaviour due to thermal deformations of the CMM structure in a general sense. Hence, the denominated thermal errors are induced, detrimental to precision and repeatability of CMMs (Bryan, 1995).

\section{Nomenclature}

$C_{\text {laser }}=$ coefficient of correction of laser

$D_{\text {Calculated }}=$ gauge calculated diameter

Paper accepted April, 2005. Technical Editor: Anselmo.Eduardo Diniz.
$D_{\text {Calibrated }}=$ gauge calibrated diameter

error $_{i}=$ geometric error at any position $i$ and at any thermal state

$\operatorname{error}_{i}(p)=$ geometric error at position $i$

$E x, E y$ and $E z=$ components of volumetric error at $20^{\circ} \mathrm{C}$

$E x(p, T), E y(p, T)$ and $E z(p, T)=$ components of volumetric error at any position $i$ and at any thermal state

$k=$ coverage factor

$\operatorname{Ort}_{X Y}, \operatorname{Ort}_{X Z}$ and $\operatorname{Ort}_{Y Z}=$ orthogonality errors

$p=$ position

Pitch $_{X}$, Pitch $_{Y}$ and Pitch $_{Z}=$ angular error Pitch at axis $X, Y$ and $Z$, respectively

$\operatorname{Pos}_{X}, \operatorname{Pos}_{Y}$ and $\operatorname{Pos}_{Z}=$ positioning error at axis $X, Y$ and $Z$, respectively

Roll $_{X}$ and Roll $_{Y}=$ angular error Roll at axis $X$ and $Y$, respectively

$s=$ standard deviation

$R^{2}=$ correlation coefficient

$R x_{Y}$ and $R x_{Z}$ = straightness error of axis $X$ direction $Y$ and $Z$, respectively

$R y_{X}$ and $R y_{Z}=$ straightness error of axis $Y$ direction $X$ and $Z$, respectively

$R z_{X}$ and $R z_{Y}=$ straightness error of axis $Z$ direction $X$ and $Y$, respectively

$R_{\text {Laser }}=$ laser resolution

$R_{M M 3 C}=$ machine resolution

$T$ = temperature

$\operatorname{Verror}_{i}(T)=$ error variation at any point $i$ for $i=1, \ldots, 4$;

verror $_{i}(p, T)=$ thermally induced variation of geometric error $i$ at position $p$.

$v_{\text {eff }}=$ effective degree of freedom

$X, Y$ and $Z=$ coordinates

$Y_{34}, X_{23}, Z_{12}$ and $Z_{45}=$ fixed offset

$Y_{a w_{X}}, \operatorname{Yaw}_{Y}$ and $\mathrm{Yaw}_{Z}=$ angular error Yaw to axis $X, Y$ and $Z$, respectively

\section{Greek Symbols}

$\alpha_{E}$ - coefficient of thermal expansion of the scale

$\beta_{1}, \ldots, \beta_{n}=$ least squares estimators

$\gamma_{1}, \ldots, \gamma_{j}=$ least squares estimators

$\Delta T=$ difference between the room temperature and the reference temperature 
$\delta T=$ difference between the scale temperature and the reference temperature

\section{Subscripts And Superscripts}

$i=$ positions where thermal drift was observed

$j=$ thermocouples

\section{Thermal Errors. General Aspects}

Several studies have been developed with the aim of understanding the characteristics, magnitudes and sources of thermally induced errors so that their effects can be minimized. If the works concerning thermal errors were chronologically analysed, one would have noticed they have been published for 40 years. Despite all the efforts, little progress was observed until the mideighties, when there was a substantial intensification of the number of publications, especially on error compensation. However, the attained progress is still distant from ideal. Thermal errors, their costs and sources have remained practically unchanged (Bryan, 1995).

Bryan (1967) assessed the state of the art and relevance of thermally induced errors. According to Bryan, errors due to temperature variation either present the same magnitude or are greater than kinematic, static and dynamic errors. Thermally induced errors are responsible for a considerable fraction of the total error of a machine tool (40-70\%). Therefore, the economic implication of thermal errors is eminent.

Bryan (1990) presented an evaluation of the achieved advance from 1967 to 1990 . In this occasion, the author denoted that the problem related to thermal errors is one of the most important on dimensional metrology and precision engineering. In general, thermal deformations have more influence on the precision of machined parts than the mechanical stiffness of the machine.

Ramesh et al (2000) published a paper about thermal errors in machine tools that basically discusses the work concerning the study, measurement, modelling and compensation of thermally induced errors that were produced in the nineties. The authors emphasize that, despite the advances, the problem regarding thermal influences in machine tools remains unsolved, challenging the international scientific community.

Regarding coordinate measuring machines, the thermal influences issue remains even more critical, due to the poor availability of research on the theme and the insipience of the published results. The subject, although not recent, remains contemporary.

\section{Methods and Techniques Employed to Derive Equations for the Thermal Errors}

Numerous studies were developed with the aim to investigate the sources, characteristics, magnitudes and behaviour of thermally induced errors. Among the mathematical means that were used to describe the behaviour of geometrical errors, the following deserve close observation: Homogeneous Transformations, Finite Element Technique and Statistical Methods, being the homogeneous transformations the most used. Subsequently, some works are mentioned to assert the vast and the potential utilization of homogeneous transformations.

Donmez et al (1986) employed homogeneous transformations on the modelling of machine tools for the determination of the spatial relationship between tool and workpiece. The methodology was applied to a turning machine. On the proposed formulation, the error was written as the sum of two polynomials. The first one expressed the variation of the geometrical error as a function of the moving carriage position, while the second described the error variation as a function of temperature, that is, a thermally induced error. The coefficients of the model were determined by means of the least squares method. The proposed equations can be modified and applied to other types of machine tools.

Shivaswamy (1992) presented a model for a three-axis machine using homogeneous coordinates. In order to describe the model, a carefully conduced survey of the geometrical and thermal errors was accomplished. Given the application of the model, it was possible to implement an error compensation routine.

Ferreira and $\mathrm{Liu}^{\mathrm{a}}$ (1986) proposed an analytical model aiming at the prediction of geometrical errors in machine tools. They employed homogeneous transformations matrices and presented a method to estimate the model coefficients by means of expressions for the individual errors. The model grants interesting features, since it allows the evaluation of the variation of the error components and models straightness as a function of the variation of angular errors. Additionally, the coefficients of the model can be obtained by the observation of the error array over a few points (nine) in the working space of the machine.

The work of Ferreira and Liu ${ }^{\mathrm{b}}$ (1986) presents an application of the model described by Ferreira and Liu ${ }^{\mathrm{a}}$ (1986) on a machine with two moving axes. The model parameters calculation was performed using measurements obtained on nine points that were distributed in the working volume of the machine. The experiment was achieved by means of the monitoring of thermocouples located on several positions on the machine structure, until the thermal steady state was reached. The machine was evaluated both in warming up and in cooling down conditions. The collected data was adequately applied to the mathematical expressions and the total error could be predicted over the entire working plane.

Kreng et al (1994) presented a model to express the error on the working volume of a three-axis machining centre. Rigid body kinematics and homogeneous coordinate transformations were employed in the modelling process. The error model coefficients were estimated from measurements on ten locations distributed through the working volume. The model could not adequately predict the cold machine error, but presented good results during the warming up period, where $75 \%$ of the errors around $100 \mu \mathrm{m}$ of magnitude were predicted. Among the possible reasons to justify the inefficiency of the model to describe errors of the cold machine according to the authors, remains the poor probing system accuracy and the mechanical artefact used to collect thermal drift values, besides the inadequate error modelling by means of a quadratic approximation.

Pereira (1995) elaborated a model for an NC cylindrical grinding machine, by means of homogeneous transformations, considering the influence of thermal gradients acting on the machine. The model described the movement of the elements of the machine and the respective errors. The model allowed for the total error survey considering thermal influences. As a conclusion, the significant influence of temperature variations on the behaviour of almost all individual errors of the machine is remarkable.

Vieira Sato (1998), using homogeneous transformations, developed the kinematical model of an NC cylindrical grinding machine, considering the influences of thermal variations. The results synthesized with the model were evaluated, discussed and compared to the errors measured on the workpieces produced by the machine. An excellent ability of the model to predict the grinding machine planar error was observed. From the proposed model, at least $80 \%$ of the dimensional errors could be corrected.

Wang et al (1998) employed homogeneous transformations to model and study the behaviour of a three-axis machining centre. In order to develop their work, the authors assumed that each axis presents six errors: one positioning error, two straightness errors and 
three angular errors. Therefore, 21 sources of errors were studied, including three orthogonal ones.

Yuan and $\mathrm{Ni}$ (1998) developed a general procedure for geometric error and thermal error compensations, as well as the ones induced by cutting forces. The geometric and thermal error synthesization model is based on the presumption of rigid body. The volumetric error array for any position was determined by means of homogeneous transformations.

\section{Development of the Error Synthesization Model}

This work presents a mathematical formulation to obtain the equations for the volumetric error components in coordinate measuring machine considering thermal influences. All experimental runs for the acquisition of error and temperature data were conducted on a moving bridge CMM. The machine consists of a cast aluminum structure with the shape of a bridge that moves with relation to a granite flat surface. The workpieces are attached to the flat surface by means of screws, clamps and fixtures. The flat surface is mounted on balls over vee-blocks on the steady structure of the machine. Three sets of aerostatic bearings provide the movement of axes $X, Y$ and $Z$ over the slideways. The bearings require dry and clean compressed air to produce the layer that sustains the moving parts of the structure.

The heat sources that affect the performance of the evaluated machine are: compressed air; thermal memory; environment; illumination system; individuals and other machines. On the evaluated CMM, there is a reduced number of heat sources, as well as the internally generated heat. However, the thermal influence problem is not simple, due to the demanding precision requirements for the CMM

Using an error synthesization model obtained by means of homogeneous transformations, each component of the volumetric error can be described as the sum of different parts that are related to the geometric errors of the machine and to the corresponding Abbè offsets. Geometric errors were described as functions of position and temperature. The proposed model is based on the straightforwardness of application and adaptation of the homogeneous transformations to any kind of CMM and on the efficient diagnosis ability of the error synthesization method.

The modelling was carried out in two stages. Firstly, the equations of the volumetric error for a reference temperature of $20^{\circ} \mathrm{C}$ were determined by means of homogeneous transformations. The detailed description of the model can be observed in Sosa Cardoza (1995) and Souza (2000). Next, the equations of the geometric errors thermally induced variations were determined using regression techniques and the least square method.

Hence, the equations of the volumetric error components are given in (1), (2) and (3).

$$
\begin{aligned}
E x & =\text { Pos }_{X}+R y_{X}+R z_{X}+\left[\text { Ort }_{X Y}+\mathrm{Yaw}_{X}\right] \cdot Y_{34}+ \\
& +\left[\text { Ort }_{X Z}+\text { Pitch }_{X}+\text { Yaw }_{Z}+\text { Roll }_{Y}\right]\left(-Z-Z_{45}\right)+\text { Roll }_{Y} \cdot Z_{12} \\
E y= & \text { Pos }_{Y}+R x_{Y}+R z_{Y}+\left[\text { Ort }_{X Y}+\text { Yaw }_{Y}\right] \cdot\left(X_{23}+X\right)-\text { Pitch }_{Y} \cdot Z_{12}+ \\
& -\left[\text { Ort }_{Y Z}+\text { Roll }_{X}+\text { Pitch }_{Y}+\text { Pitch }_{Z}\right]\left(-Z-Z_{45}\right) \\
& E z=\text { Pos }_{Z}+R x_{Z}+R y_{Z}-\text { Roll }_{Y} \cdot\left(X_{23}+X\right)-\left[\text { Roll }_{X}+\text { Pitch }_{Y}\right] \cdot Y_{34}
\end{aligned}
$$

Besides the error values and Abbè offsets at $20^{\circ} \mathrm{C}$, it is necessary to know the magnitude of the variation experienced by the errors and steady offsets due to temperature variation. Therefore, geometric errors must be changed for different thermal states. Resulting data must be treated as functions of position and temperature and finally must be adequately introduced in the equations of synthesization.

Thus, each geometric error was written as the sum of two parts Eq. (4). The first one represents the geometric error at $20^{\circ} \mathrm{C}$ and only depends on the position of the corresponding carriage. The second part represents the thermally induced error variation. It can be described as a function of temperature and position, since temperature variation may cause irregularly distributed error variation along the coordinate axes.

$$
\operatorname{error}_{i}=\operatorname{error}_{i}(p)+\operatorname{verror}_{i}(p, T)
$$

where error $_{i}$ is the error at any position $i$ and at any thermal state, $\operatorname{error}_{i}(p)$ is the geometric error $i$ at position $p$ and $\operatorname{verror}_{i}(p, T)$ is the thermally induced variation of geometric error $i$ at position $p$.

The mathematical determination of geometric error variation due to changes in temperature is rather complex. Consequently, collected data from thermal drift at four points distributed along each evaluated axis were employed. One data set was acquired at each observation point. These sets represent the thermally induced variation of the error at a given position, until steady state. Expressions describing error variation as a function of temperature at each drift observation point were obtained by means of regression techniques. The variation can be written as:

$$
\operatorname{Verror}_{i}(T) \cong \gamma_{0}+\gamma_{1} T_{1}+\gamma_{2} T_{2}+\ldots+\gamma_{j} T_{j}
$$

where $\operatorname{Verror}_{i}(T)$ is the error variation at any point $i$ for $i=1, \ldots, 4$;

$T$ contains the components of the array of temperature variation from the several thermocouples $j$, for every $j=1, \ldots, 18$.

In the temperature variation array, only data from thermocouples whose temperature was considered as significantly influent were included. The selection of values that compose the temperature array at each point $i$ was performed by means of a stepwise procedure. Therefore, thermocouples whose temperature presented a correlation greater than or equal to $99.9 \%$ were grouped. Subsequently, the effects of variables $p$ and $T$ were superposed for the determination of error variation at any position and at any thermal state, using regression techniques and the least squares method. where $\beta_{i}$, $(i=0$, $\ldots, n)$ are the regression coefficients; $p_{i},(i=1, \ldots, 4)$ are the positions where thermal drift was observed.

$$
\operatorname{verror}_{i}(p, T)=\beta_{0}(T)+\beta_{1}(T) \cdot p_{i}+\beta_{2}(T) \cdot p_{i}^{2}+\ldots+\beta_{n}(T) \cdot p_{i}^{n}
$$

The estimation of coefficients $\beta_{n}$ was made possible setting an equality relationship between Eq. (6) and error array given by (5) and applying the least squares method. The resulting system is given by (7). Least squares estimators $\beta_{0}, \beta_{1}, \beta_{2}, \ldots, \beta_{n}$ are determined so as to minimize Eq. (8).

$$
\begin{gathered}
\left\{\begin{array}{l}
\beta_{0}(T)+\beta_{1}(T)^{*} p_{1}+\beta_{2}(T)^{*} p_{1}^{2}+\ldots+\beta_{n}(T)^{*} p_{1}^{n}=\operatorname{Verror}_{1}(T) \\
\beta_{0}(T)+\beta_{1}(T)^{*} p_{2}+\beta_{2}(T)^{*} p_{2}^{2}+\ldots+\beta_{n}(T)^{*} p_{2}^{n}=\operatorname{Verror}_{2}(T) \\
\beta_{0}(T)+\beta_{1}(T)^{*} p_{3}+\beta_{2}(T)^{*} p_{3}^{2}+\ldots+\beta_{n}(T)^{*} p_{3}^{n}=\operatorname{Verror}_{3}(T) \\
\beta_{0}(T)+\beta_{1}(T)^{*} p_{4}+\beta_{2}(T)^{*} p_{4}^{2}+\ldots+\beta_{n}(T)^{*} p_{4}^{n}=\operatorname{Verror}_{4}(T)
\end{array}\right. \\
S\left(\beta_{0}, \beta_{1}, \ldots, \beta_{n}\right)=\sum_{i=1}^{m} \varepsilon_{i}^{2}=\sum_{i=1}^{m}\left(\operatorname{Verrok}_{i}-\beta_{0}-\beta_{1} x_{1 i}-\ldots-\beta_{n} x_{n i}\right)^{2}
\end{gathered}
$$

Estimators in Equation (8) were obtained by means of commercial software. The position and temperature effects 
superposition model is not linear and due to the complexity of the terms, they are not printed.

\section{Calibration of the Machine}

Once the modelling strategy was defined, the experiments required for data acquisition were adequately planned and executed. The experiments consist of geometric errors survey, acquisition of drift values and temperature monitoring. Data referring to positioning geometric errors, straightness and angular errors pitch and yaw from all axes were obtained with a Hewlett-Packard HP5529A Laser interferometric system. A mechanical square and a LVDT type transducer were used to provide for the determination of the orthogonal errors. A Rank Taylor-Hobson Talyvel 3 was employed on the determination of roll errors on axis $X$ and $Y$.

In order to observe temperature, 28 copper-constantan T-type thermocouples were employed. Sixteen thermocouples were placed on several locations on the structure of the CMM, three were dedicated to the monitoring of local heat sources and eight were used to monitor spatial thermal gradients. A reference thermocouple was set into a mixture of water and ice. Similar arrangements were used by Pereira (1995), Vieira Sato (1998) and Kruth et al (2001).

Thermocouples designated to verify environmental temperature were placed at a wooden block due to the thermal insulation that wood provides. The copper sheets at the end of each thermocouple were adhered to a $31 \mathrm{~mm}$ diameter, $8 \mathrm{~mm}$ thick aluminum disc for thermal inertia.

A 32-channel multiplexer was built to allow temperature data acquisition from the thermocouples. A 12-bit resolution A/D converter data acquisition board was employed to convert analogue voltage signals from each thermocouple into digital values. Moreover, an algorithm was developed in Delphi language to collect data from the thermocouples.

The drift test developed in this work consisted of the observation of the geometric errors variation at a given position until stabilization. The test was necessary to define the correlation between error variation and temperature variation.

Taking the work by Vieira Sato (1998) as reference, data referring to error variation at four locations distributed along the evaluated axis were collected. Both temperature stabilization and acquisition time of thermally induced error variation data was of 3h10min. A temperature gradient was introduced, which varied from $20^{\circ} \mathrm{C}$ to $26^{\circ} \mathrm{C}$ during the warming up period and from $26^{\circ} \mathrm{C}$ to $20^{\circ} \mathrm{C}$ at cooling down. As a result, four data sets that describe error variation at each observation point were obtained.

\section{Experimental Results}

Results concerning the verification of spatial thermal gradients, temperature distribution upon the CMM structure, stabilization time, analysis and discussion of the geometric errors survey are presented as follows. During the analysis of temperature data sets collected from the right hand side of the $\mathrm{CMM}$, the existence of a vertical gradient varying from $0.4^{\circ} \mathrm{C} / \mathrm{m}$ and $1^{\circ} \mathrm{C} / \mathrm{m}$ was detected. Thermocouples near the ceiling of the room registered higher temperatures. At the left hand side of the CMM, vertical gradients for warming up and cooling down were up to $2.6^{\circ} \mathrm{C} / \mathrm{m}$, approximately. In this case, the temperature of the thermocouple near the ceiling was lower due to air flow from the air-conditioning system.

A graph containing the curves that describe the behaviour of axis $\mathrm{Y}$ positioning errors at $20^{\circ} \mathrm{C}$ is presented in Fig. 1. In this graph, the four curves correspond to positioning errors for forward and backward motion and standard deviation of errors for forward motion. Curves for standard deviation for backward motion are very similar to the curves for forward motion.

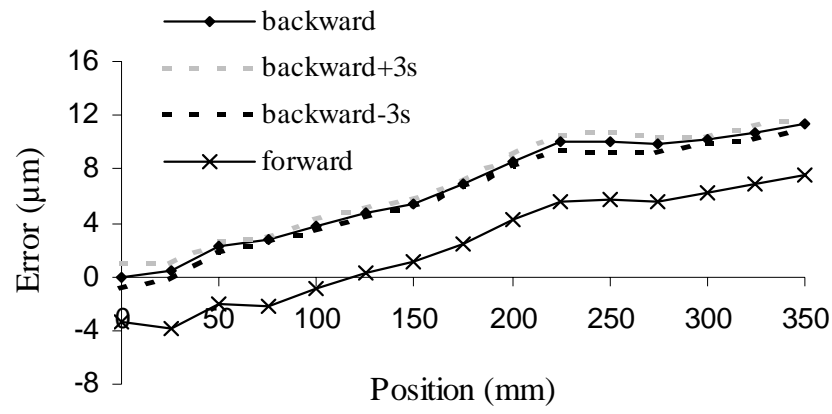

Figure 1. Axis $\mathrm{Y}$ positioning error at $20^{\circ} \mathrm{C}$.

It can be observed in Fig. 1 that axis $Y$ positioning error is positive with increasing tendency and hysteresis of about $3.7 \mu \mathrm{m}$, staying almost constant along axis $Y$. Interval of \pm 3 s is small.

The graph in Fig. 2 presents the curves that describe thermally induced variation of positioning error $Y$.

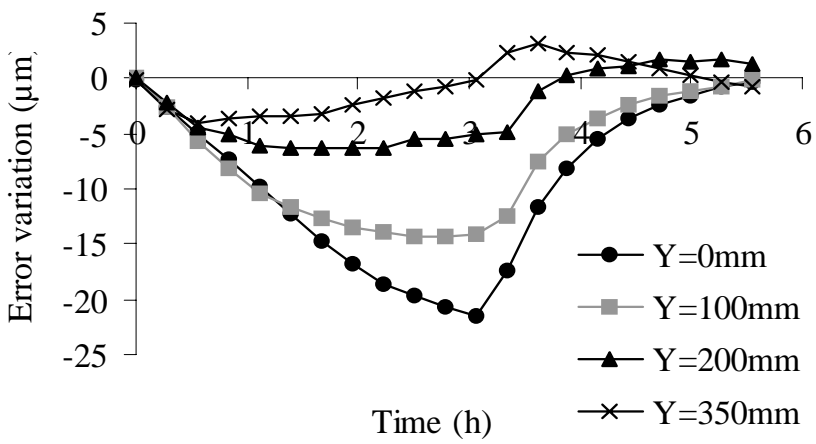

Figure 2. Axis $\mathrm{Y}$ positioning error drift curves.

In Fig 2, it can be observed that at certain positions, in the early beginning of cooling down process, error variation presents an increasing tendency. This fact is ascribed to system inertia and to the environmental conditions in the room at the moment of data acquisition. If humidity is high, heating system will work harder, causing cyclic temperature elevations.

Equations (9), (10), (11) and (12) describe axis $Y$ positioning error variation as a function of temperature in the respective observation points.

$$
\begin{aligned}
\operatorname{Verrog}(T)= & -3.50 \cdot T_{1}+19.60 \cdot T_{2}-14.00 \cdot T_{4}-8.50 \cdot T_{5}+8.76 \cdot T_{7}+ \\
& +3.20 \cdot T_{8}-1.20 \cdot T_{9}-2.30 \cdot T_{10}+7.38 \cdot T_{11}-3.00 \cdot T_{12}- \\
& -2.10 \cdot T_{13}+2.90 \cdot T_{14}-5.50 \cdot T_{15}-2.30 \cdot T_{16}
\end{aligned}
$$

$\operatorname{Verror}_{5}(T)=11.70 \cdot T_{2}-1.70 \cdot T_{3}+2.75 \cdot T_{4}-7.10 \cdot T_{6}-3.80 \cdot T_{7}+$ $+0.87 \cdot T_{8}-0.97 \cdot T_{9}-0.85 \cdot T_{10}+1.46 \cdot T_{11}-1.80 \cdot T_{12}-$ $-1.70 \cdot T_{14}-2.70 \cdot T_{15}-4.70 \cdot T_{16}$

$\operatorname{Verroko0}_{20}(T)=12.30 \cdot T_{2}-1.80 \cdot T_{3}-3.80 \cdot T_{4}-1.90 \cdot T_{5}+1.35 \cdot T_{7}-$ $-3.40 \cdot T_{11}-2.40 \cdot T_{12}-1.30 \cdot T_{16}$ 


$$
\begin{aligned}
\operatorname{Verrog}_{00}(T) & =4.02 \cdot T_{1}+5.03 \cdot T_{2}+1.79 \cdot T_{3}+1830 \cdot T_{4}-5.20 \cdot T_{6}+ \\
& +14.00 \cdot T_{7}-9.10 \cdot T_{8}+1.74 \cdot T_{11}-1.80 \cdot T_{12}-5.50 \cdot T_{13}- \\
& -1.30 \cdot T_{14}+3.75 \cdot T_{15}+1.80 \cdot T_{17}
\end{aligned}
$$

Correlation coefficients for each curve were 99.89\%, 99.83\%, $99.7 \%$ and $99.8 \%$ respectively. Analysis of residuals showed random behaviour, following an approximately normal distribution with mean value close to zero and constant variance.

The expression for the variation of axis $Y$ positioning error was obtained through the sum of the solution of system (13) with the error at reference state, so that the function describing the behaviour of the referring error was obtained.

$$
\left\{\begin{array}{l}
\operatorname{verror}(0, T)=\operatorname{Verror}_{0}(T) \\
\operatorname{verror}(75, T)=\operatorname{Verror}_{75}(T) \\
\operatorname{verror}(200, T)=\operatorname{Verror}_{200}(T) \\
\operatorname{verror}(300, T)=\operatorname{Verror}_{300}(T)
\end{array}\right.
$$

An analysis performed on the results obtained during the positioning thermally induced error variations for all axes allowed for the conclusion that the assembly principle between slide and scale influences magnitude and behaviour of these errors when the machine is submitted to environmental temperature variation.

In the evaluated machine, the scale is glued to the slide in such a manner that the scale is able to freely expand and contract from a point that is very close to its centre. Thus, the smaller errors were found when workpieces to be measured were positioned at the central region of the reference flat surface of the machine. Furthermore, when the scale is glued to the slide, the differential expansion error is reduced by approximately 50\%, if compared to other assembly manners, wherein the scale is fastened at one of its ends.

Figures 3 shows straightness errors in axis $Y$. It can be observed that error values are smaller than $2 \mu \mathrm{m}$. Hysteresis is small and random errors vary within the range of $\pm 1.5 \mu \mathrm{m}$. Figure 4 shows that the behaviour of thermally induced variation on axis $Y$ straightness error is independent of observation position. Therefore, it was possible to elaborate a model for these errors using a unique thermal drift dataset raised at only one position. Similar results were obtained for axes $X$ and $Z$ (not presented).

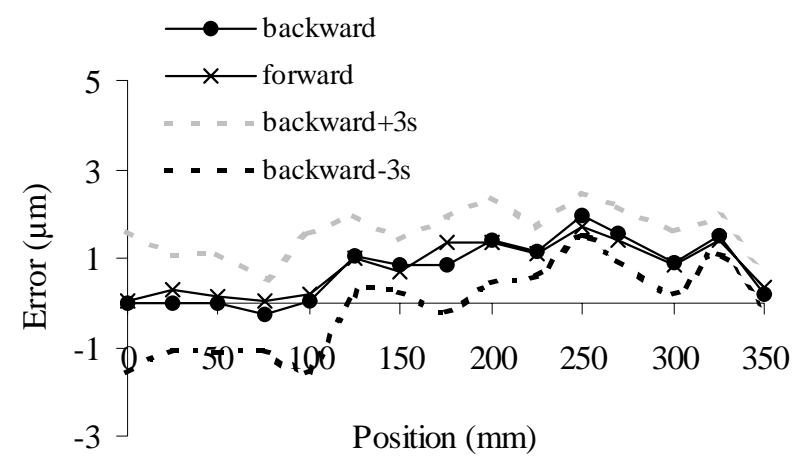

Figure 3. Axis $\mathrm{Y}$ straightness error in $\mathrm{X}$ direction, at $20^{\circ} \mathrm{C}$.

Equations (14) describe the variation of axis $Y$ straightness error in $X$ direction. Coefficient $R^{2}$ is $99.1 \%$.

$$
\begin{aligned}
V_{R Y_{X}}(T)= & -21.00 \cdot T_{2}+6.88 \cdot T_{5}+12.80 \cdot T_{6}-6.40 \cdot T_{7}-4.00 \cdot T_{8}-1.80 \cdot T_{9}+ \\
& +2.52 \cdot T_{10}-6.50 \cdot T_{12}+10.20 \cdot T_{13}+2.37 \cdot T_{15}+5.36 \cdot T_{16}
\end{aligned}
$$

Analysis of residuals showed their random behaviour, with zero mean and constant variance.

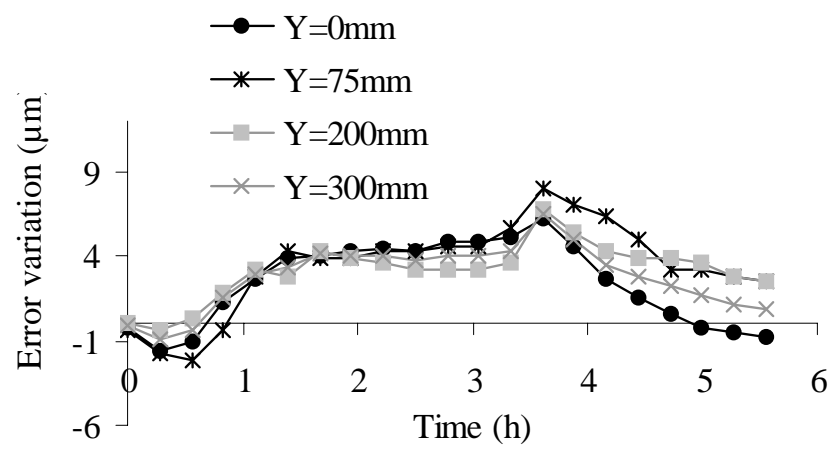

Figure 4. Curves of axis $Y$ straightness error drift in $X$ direction.

Figure 5 shows axis $\mathrm{Y}$ yaw at $20^{\circ} \mathrm{C}$. It can be observed that the error is positive and crescent, with a maximum value of approximately 7 arcseg at position $\mathrm{Y}=350 \mathrm{~mm}$. Both hysteresis and standard deviation are small.

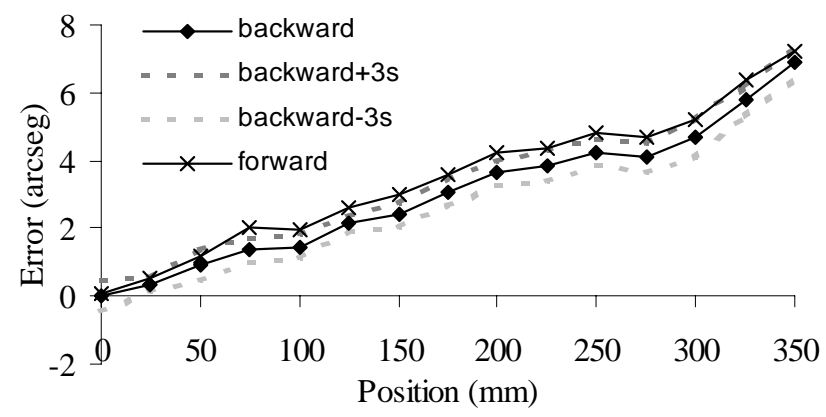

Figure 5. Axis $Y$ angular error yaw at $20^{\circ} \mathrm{C}$.

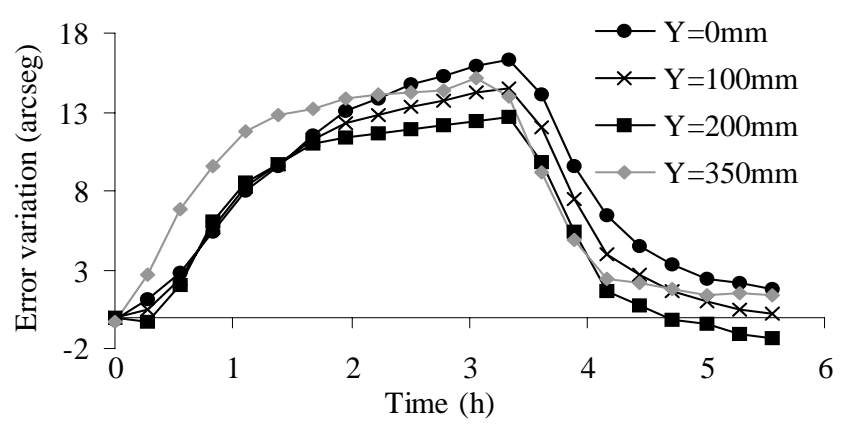

Figure 6. Axis $Y$ yaw drift curves.

Fig. 6 shows curves that describe axis $Y$ thermally induced variation of angular error yaw. It must be highlighted that magnitude and behaviour of axis $Y$ yaw error are similar at all four observation points. Similar results were observed for all angular errors yaw, pitch and roll at all axes. Thus, these errors can be obtained from data recorded at one observation position only. More information can be found in Valdés 2003.

Equation (15) was obtained by regression techniques. Correlation coefficient for the curve above was 99.86\%. Residuals presented proper behaviour so as to ensure adequacy between function and experimental data. 


$$
\begin{aligned}
V_{Y a w_{Y}}(T)= & 2.81 \cdot T_{1}-21.00 \cdot T_{2}+9.18 \cdot T_{4}+8.99 \cdot T_{5}+3.37 \cdot T_{6}-7.30 \cdot T_{7}- \\
& -2.50 \cdot T_{8}+1.35 \cdot T_{9}+1.90 \cdot T_{10}-3.30 \cdot T_{11}+1.53 \cdot T_{13}+2.80 \cdot T_{14}+ \\
& +5.01 \cdot T_{15}+3.00 \cdot T_{16}+0.57 \cdot T_{17}
\end{aligned}
$$

The results of orthogonal errors measurement at $20^{\circ} \mathrm{C}$ are $\operatorname{Ort}(x y), \operatorname{Ort}(x z)$ and $\operatorname{Ort}(y z)$ 10.31, 20.63 and -185.64arcseg, respectively. Values of the orthogonal errors remained constant during thermal transients.

\section{Evaluation of the Proposed Model}

The collected data sets were adequately introduced in the mathematical equations to allow for the development of the proposed model. The geometric-thermal CMM model is given by Eq. (16), (17) and (18). In these equations, a number of parcels corresponding to the thermally induced variation of some geometric errors were disregarded, as they represent the same effect as the variation of other errors.

$$
\begin{aligned}
& E x(p, T)=P \operatorname{Pos}_{X}+\delta\left[\operatorname{Pos}_{X}(p, T)\right]+R y_{X}+\delta\left[R y_{X}(T)\right]+R z_{X}+ \\
& +\left[\operatorname{Ort}_{X Y}+\operatorname{Yaw}_{X}+\delta \operatorname{Yaw}_{X}(T)\right] \cdot\left(Y_{34}+\delta Y_{34}\right)+ \\
& +\left[\begin{array}{l}
\operatorname{Ort}_{X Z}+\operatorname{Pitch}_{X}+\delta\left[\operatorname{Pitch}_{X}(T)\right]+\operatorname{Yaw}_{Z} \\
+\delta\left[\operatorname{Yaw}_{Z}(T)\right]+\operatorname{Roll}_{Y}+\delta\left[\operatorname{Roll}_{Y}(T)\right]
\end{array}\right] \cdot\left[-Z-\left(Z_{45}+\delta Z_{45}\right)\right]+ \\
& +\left\{\operatorname{Roll}_{Y}+\delta\left[\operatorname{Roll}_{Y}(T)\right]\right\} \cdot\left(Z_{12}+\delta Z_{12}\right) \\
& E y(p, T)=P o s_{Y}+\delta\left[\operatorname{Pos}_{Y}(p, T)\right]+R x_{Y}+R z_{Y}+\delta\left[R z_{Y}(T)\right] \\
& +\left[\operatorname{Ort}_{X Y}+\operatorname{Yaw}_{Y}+\delta\left[\operatorname{Yaw}_{Y}(p, T)\right] \cdot\left(X_{23}+\delta X_{23}+X\right)-\right. \\
& -\left[\text { Pitch }_{Y} \cdot \delta\left[\text { Pitch }_{Y}(p, T)\right]\right] \cdot\left(Z_{12}+\delta Z_{12}\right)- \\
& -\left[\begin{array}{l}
\operatorname{Ort}_{Y Z}+\operatorname{Roll}_{X}+\delta\left[\operatorname{Roll}_{X}(p, T)\right]+\operatorname{Pitch}_{Y}+ \\
+\delta\left[\operatorname{Pitch}_{Y}(p, T)\right]+\operatorname{Pitch}_{Z}+\delta\left[\operatorname{Pitch}_{Z}(p, T)\right]
\end{array}\right] \cdot\left[-Z-\left(Z_{45}+\delta Z_{45}\right)\right] \\
& E z=P o s_{Z}+\delta\left[\operatorname{Pos}_{Z}(p, T)\right]+R x_{Z}+\delta\left[R x_{Z}(T)\right]+R y_{Z}- \\
& -\left[\operatorname{Roll}_{Y}+\delta\left[\operatorname{Roll}_{Y}(p, T)\right]\right] \cdot\left(X_{23}+\delta X_{23}+X\right)- \\
& -\left[\operatorname{Roll}_{X}+\delta\left[\operatorname{Roll}_{X}(p, T)\right]+\operatorname{Pitch}_{Y}+\delta\left[\operatorname{Pitch}_{Y}(p, T)\right]\right] \cdot\left(Y_{34}+\delta Y_{34}\right)
\end{aligned}
$$

The validation of the obtained model was accomplished by measuring a ring gauge. Thus, values of the diameters measured with the CMM were compared to the ones obtained by the synthesization model.

The ring gauge was measured at several positions and at different temperatures. The coordinates of 11 randomly distributed points on the surface that defines the ring diameter were collected. The values of the components of the volumetric error were synthesized at the coordinates of the collected points by means of the proposed model. Next, the error correction at the coordinates of the measured points was performed, and the corrected coordinate values were obtained. Subsequently, the diameter was estimated and the difference between calculated and standard diameter values was determined. Finally, the difference between calculated and standard diameter values was determined. Standard diameter was obtained by means of calibration. In order to perform the task, a universal measuring machine made by Societè Genevoise D'Instruments de Physique (SIP) model 302M was employed. The ring gauge calibrated diameter was $181.0124 \mathrm{~mm} \pm 1 \mu \mathrm{m}$ at $20^{\circ} \mathrm{C}$, whereas at $26^{\circ} \mathrm{C}$, the value was $180.0137 \mathrm{~mm} \pm 0.7 \mu \mathrm{m}$.

Having known calculated and calibrated diameters, residual error difference was determined.

$$
\text { Residual Error }=D_{\text {Calculated }}-D_{\text {Calibrated }}
$$

From residual error values, an analysis was executed to decide whether the model is adequate or not. Model adequacy is conditioned to residual error values relatively small and normally distributed.

\section{Comparison Between Errors Found on Workpiece Measurement and Modelled}

Initially, results referring to geometric model evaluation of the CMM are presented. Fig. 7 shows values of errors found on the measurement process of the ring gauge diameter at various positions, at $20^{\circ} \mathrm{C}$. For the coordinates of the measurement points, volumetric error components values were synthesized and then compensated. Difference between measurement and synthesized errors consists of the residual error shown in Fig. 7.
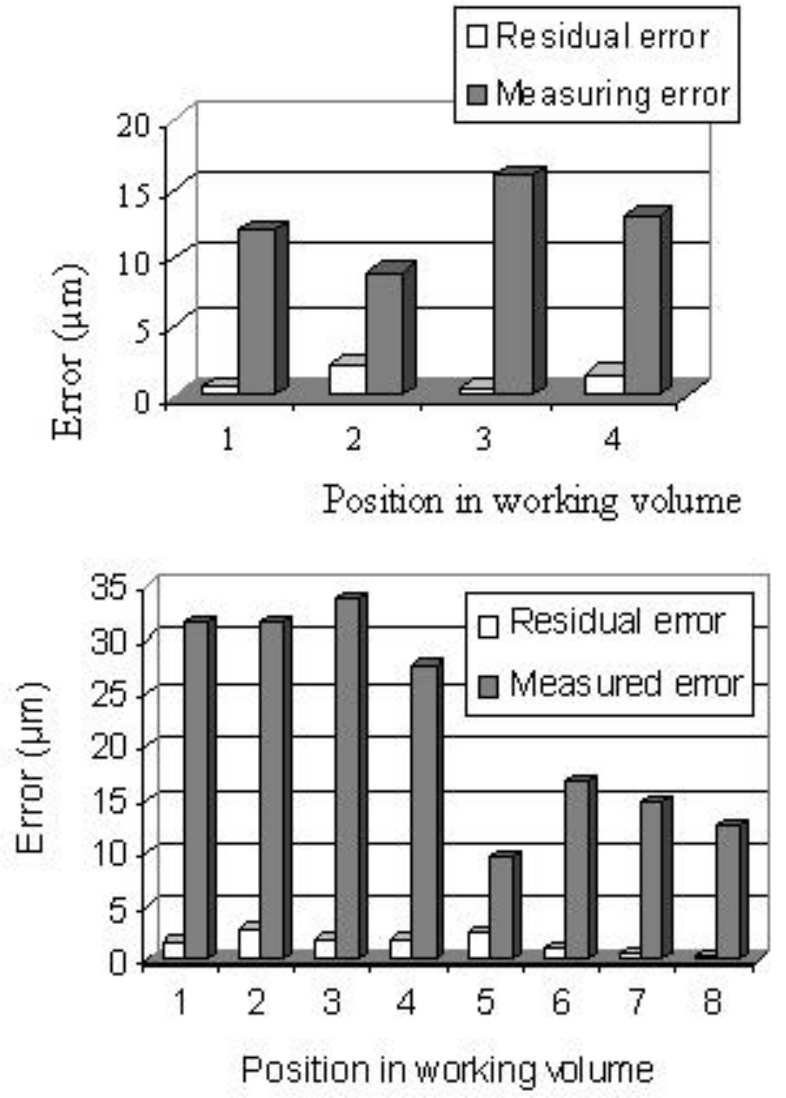

Figure 7. Measurement and residual errors at 20 (left) and $26^{\circ} \mathrm{C}$ (right).

In Fig. 7, (left) it can be verified that errors found during measurement of ring diameter at $20^{\circ} \mathrm{C}$, i.e., the difference between measured and calibrated values was smaller than $16 \mu \mathrm{m}$ in every case, whereas errors after compensation were not greater than $2.3 \mu \mathrm{m}$. The geometric model allowed for a reduction on measurement errors of at least $74 \%$. These results can be considered adequate. Table 1 shows the values of measured and synthesized diameters, using the geometric model of the machine, as well as the residual errors and error reduction percentage with respect to calibrated values. 
Table 1. Measured and synthesized diameters.

\begin{tabular}{|c|c|c|c|c|c|}
\hline Position & $\begin{array}{c}\text { Temperature } \\
\left({ }^{0} \mathrm{C}\right)\end{array}$ & $\begin{array}{c}\text { Diameter. } \\
\text { measured } \\
(\mathrm{mm})\end{array}$ & $\begin{array}{c}\text { Diameter. } \\
\text { modelled } \\
(\mathrm{mm})\end{array}$ & $\begin{array}{c}\text { Residual } \\
\text { error } \\
(\mu \mathrm{m})\end{array}$ & Percentage \\
\hline 1 & 20 & 180.000 & 180.0126 & 0.60 & 94.96 \\
\hline 2 & 20 & 180.003 & 180.0143 & 2.26 & 74.85 \\
\hline 3 & 20 & 179.996 & 180.0115 & -0.52 & 96.74 \\
\hline 4 & 20 & 179.999 & 180.0136 & 1.59 & 87.77 \\
\hline
\end{tabular}

Figure 7 (right) shows results obtained during evaluation of the model at $26^{\circ} \mathrm{C}$. It can be observed that thermal-geometric errors at positions 1-4 are much greater than geometric errors on Figure 7. This fact reinforces the importance of the effect of temperature variation on measurement results. Residual errors at all positions were small, being less than $3 \mu \mathrm{m}$. At positions 1-4, close to the reference flat surface, errors were reduced by at least $90 \%$. At positions 5-8, where errors are smaller, correction was greater than $74 \%$.

Table 2 shows the values of measured diameters and the ones synthesized by means of the proposed thermal-geometric model, as well as the residuals and error reduction percentage.

Table 2. Results of the thermal-geometric model evaluation at $26{ }^{\circ} \mathrm{C}$.

\begin{tabular}{|c|c|c|c|c|c|}
\hline Position & $\begin{array}{c}\text { Temperature } \\
\left({ }^{0} \mathrm{C}\right)\end{array}$ & $\begin{array}{c}\text { Diameter } \\
\text { measured } \\
(\mathrm{mm})\end{array}$ & $\begin{array}{c}\text { Diameter } \\
\text { modelled } \\
(\mathrm{mm})\end{array}$ & $\begin{array}{c}\text { Residual } \\
\text { error } \\
(\mu \mathrm{m})\end{array}$ & Percentage \\
\hline 1 & 26 & 179.982 & 180.0119 & -1.81 & 94.28 \\
\hline 2 & 26 & 179.982 & 180.0107 & -2.96 & 90.66 \\
\hline 3 & 26 & 179.980 & 180.0116 & -2.11 & 93.72 \\
\hline 4 & 26 & 179.986 & 180.0117 & -2.02 & 92.71 \\
\hline 5 & 26 & 180.004 & 180.0112 & -2.49 & 74.26 \\
\hline 6 & 26 & 179.997 & 180.0149 & 1.17 & 92.98 \\
\hline 7 & 26 & 179.999 & 180.0129 & -0.76 & 94.8 \\
\hline 8 & 26 & 180.001 & 180.0140 & 0.34 & 97.26 \\
\hline
\end{tabular}

At $20^{\circ} \mathrm{C}$, standard deviation of measurements is $2.8 \mu \mathrm{m}$. After error correction, the deviation becomes $1.6 \mu \mathrm{m}$. Dispersion after compensation is more compact at $26^{\circ} \mathrm{C}$.

From the measured diameters, it could be verified that when the ring gauge was placed at the centre of the reference flat surface of the CMM, thermally induced errors were smaller. These results were accredited to the construction principle of this machine. The scales were glued to the slides permitting their expansion from some point near their centres.

Table 3 shows the values for the ring diameter, measured during the machine warm-up, from $20^{\circ} \mathrm{C}$ to $26^{\circ} \mathrm{C}$, respectively. The fifth column (drift) corresponds to the value of the thermally induced error.

At position 9, at the centre of the flat surface, thermally induced variation of diameter values was $50 \%$ smaller than at other positions and it confirms that smaller errors occur when workpieces are placed at the centre of the flat surface.

Table 3. Thermally Induced Errors.

\begin{tabular}{|c|c|c|c|c|}
\hline Position & $\begin{array}{c}\text { Temperature } \\
\left({ }^{\circ} \mathrm{C}\right)\end{array}$ & $\begin{array}{c}\text { Diameter } \\
\text { measured }(\mathrm{mm})\end{array}$ & $\begin{array}{c}\text { Error } \\
(\mu \mathrm{m})\end{array}$ & $\begin{array}{c}\text { Drift } \\
(\mu \mathrm{m})\end{array}$ \\
\hline 1 & 20 & 180.000 & 12 & \\
\hline 2 & 20 & 180.003 & 9 & \\
\hline 3 & 20 & 179.996 & 16 & \\
\hline 4 & 20 & 179.999 & 13 & \\
\hline 9 & 20 & 180.003 & 9 & \\
\hline 1 & 26 & 179.982 & 32 & 20 \\
\hline 2 & 26 & 179.982 & 32 & 23 \\
\hline 3 & 26 & 179.980 & 34 & 18 \\
\hline 4 & 26 & 179.986 & 28 & 15 \\
\hline 9 & 26 & 179.998 & 16 & 7 \\
\hline
\end{tabular}

\section{Estimation of Uncertainty Associated to Volumetric Error Components}

Finally, estimation of the measurement uncertainty associated to the components of volumetric error was performed. In order to accomplish the task, the law of uncertainty propagation was applied to the equations of the machine thermal-geometric model, in accordance with the Guide to the expression of uncertainty in measurement (ISO GUM 1995).

Table 4. Uncertainty of axis X275 positioning error at $20^{\circ} \mathrm{C}$.

\begin{tabular}{|c|c|c|c|c|c|}
\hline $\begin{array}{l}\text { Source of } \\
\text { uncertainty }\end{array}$ & $\begin{array}{l}\text { Uncertainty } \\
\text { type }\end{array}$ & $\begin{array}{l}\text { Probability } \\
\text { distribution }\end{array}$ & $\begin{array}{l}\text { Sensitivity } \\
\text { coefficient }\end{array}$ & $\begin{array}{l}\text { Degrees of } \\
\text { freedom. }\end{array}$ & $\begin{array}{c}\text { Standard } \\
\text { uncertaint } \\
\mathrm{y}(\mu \mathrm{m})\end{array}$ \\
\hline $\mathrm{M}$ & A & Normal & $1 \mu \mathrm{m}$ & 4 & 0.074 \\
\hline $\mathrm{R}_{\mathrm{MM} 3 \mathrm{c}}$ & B & Rectangular & $1 \mu \mathrm{m}$ & $\infty$ & $1.15 \mathrm{E}-6$ \\
\hline $\mathrm{R}_{\text {laser }}$ & B & Rectangular & $1 \mu \mathrm{m}$ & $\infty$ & 0.0058 \\
\hline$\alpha_{\mathrm{E}}$ & B & Rectangular & $\begin{array}{c}-0.0064 \\
\mu \mathrm{m}^{0} \mathrm{C}\end{array}$ & $\infty$ & $-1.95 \mathrm{E}-10$ \\
\hline $\mathrm{C}_{\text {laser }}$ & B & Rectangular & $\begin{array}{l}0.0064 \\
\mu \mathrm{m}^{0} \mathrm{C}\end{array}$ & $\infty$ & $5.17 \mathrm{E}-7$ \\
\hline$\delta \mathrm{T}$ & B & Rectangular & $\begin{array}{l}-0.53 \mathrm{E}- \\
7 \mu \mathrm{m} /{ }^{0} \mathrm{C}\end{array}$ & $\infty$ & $-1.07 \mathrm{E}-8$ \\
\hline$\Delta \mathrm{T}$ & B & Rectangular & $\begin{array}{c}-0.139 \mathrm{E}- \\
4 \mu \mathrm{m} /{ }^{\circ} \mathrm{C}\end{array}$ & $\infty$ & $-5.17 \mathrm{E}-5$ \\
\hline \multicolumn{5}{|c|}{ Combined standard uncertainty $\left(\mathrm{u}_{\mathrm{c}}\right)$} & $0.104 \mu \mathrm{m}$ \\
\hline \multicolumn{5}{|c|}{ Effective degrees of freedom $\left(\mathrm{v}_{\text {eff }}\right)$} & 4.05 \\
\hline \multicolumn{5}{|c|}{ Coverage factor ( $\left.\mathrm{v}_{\text {eff }}, 95 \%\right)$} & $\mathrm{k}=2.78$ \\
\hline \multicolumn{5}{|c|}{ Expanded uncertainty (95 \%) } & $0.289 \mu \mathrm{m}$ \\
\hline
\end{tabular}

To estimate measurement uncertainty, it was necessary to estimate the uncertainty associated with each geometric error and offsets present in the equations of the model. Table 4 presents data referring to $X$ axis positioning error uncertainty estimation, at $20^{\circ} \mathrm{C}$.

Combined standard uncertainty was $0.104 \mu \mathrm{m}$, whereas expanded uncertainty was $0.289 \mu \mathrm{m}$. Therefore, $X$ axis positioning error measurement uncertainty at position $X=275 \mathrm{~mm}$ was $10.79 \pm 0.289 \mu \mathrm{m}$. Uncertainty values are similar to the presented ones for all positions in the machine working volume.

\section{Volumetric Error Components Associated Uncertainty}

Next, uncertainties associated to the components of the volumetric error $E x, E y$ and $E z$ were 2.43, 3.52 and $1.95 \mu \mathrm{m}$, respectively. Uncertainty values indicate that at any point in the work volume of the CMM, the components of volumetric error present uncertainty values close to $2.43,3.52$ and $1.95 \mu \mathrm{m}$, respectively. These results are slightly smaller than the ones due to the measurement strategy adopted, which considerably reduced the positioning error standard deviation. Thus, uncertainty values can be considered adequate.

\section{Conclusions}

After the evaluation and discussion of the results, the following conclusions may be considered:

Personal computer, air provided to the machine, supply cables and operator consist of localized heat sources, therefore they must be carefully located to avoid or reduce their effects. The correct manipulation of the workpieces during measurement must be emphasized.

Localized heat sources caused non-homogeneous temperature distribution in the machine structure, generating spatial thermal gradients.

The response of the machine to thermal perturbations can be considered as fast, since approximately 3 hours after the 
introduction of a temperature disturbance, thermal balance was reached. This is due to the fact that the CMM is predominantly built of aluminum.

Thermally induced variation of positioning errors depends on temperature, slide and scale materials and their principle of assembly.

It was not possible to adopt a linear model that described the thermally induced variation of positioning errors because of the nonhomogeneous temperature distribution in the machine.

The thermal behaviour of straightness and angular errors of all axes do not depend on the position of the corresponding moving carriages. Hence, during thermal drift data collection, error values can be evaluated at one point only.

Orthogonal errors do not alter their behaviour during thermal transients.

The evaluated CMM expands freely during the warming up period, since there are no restrictions to the expansion. Thus, it can be concluded that thermal effects were considered during the design and construction of the components of the machine. It can also be said that the evaluated CMM presents a correct metrological structure.

The least squares method was considered efficient to model the thermally induced variation of the positioning errors at any thermal state and position of the corresponding moving carriage.

The proposed model was efficient, allowing the reduction of volumetric errors values greater than $10 \mu \mathrm{m}$ by at least $90 \%$. For values of volumetric error smaller than $10 \mu \mathrm{m}$, reduction was greater than $75 \%$.

The proposed model was very effective for compensation of errors on the measurement of workpieces at different temperatures, despite being obtained from data sets that did not include the probing system.

It was experimentally demonstrated that magnitude of thermally induced errors, in this machine, is smaller when workpieces are located on the centre of the reference flat surface.

On most measurement positions, the parcel corresponding to thermally induced errors was up to $200 \%$ greater than the geometric errors parcel. Therefore, it was verified how temperature variations affect measurement results.

\section{Acknowledgements}

The authors are grateful to FAPESP (Fundação de Amparo à Pesquisa do Estado de São Paulo) for supporting this work. Process number 98/15436-7.

\section{References}

Bosch, J.A., 1995, “Coordinate measuring machines and systems”. New York. Marcel Dekker, Inc.

Bryan, J.B., 1967, "International status of thermal error research". Annals of the CIRP, Paris, Vol. 16, pp. 203-215.

Bryan, J.B., 1990, "International status of thermal error research". Annals of the CIRP, Paris, Vol. 39, No. 2, pp. 645-656.

Bryan. J.B., 1995, “Temperature fundamentals". In: Bosch, J.A "Coordinate measuring machines and systems". New York: Marcel Dekker. Cap. 8, pp. 227-264.

Donmez, M.A. et. al., 1986, “A General methodology for machine tool accuracy enhancement by error compensation”. Precision Engineering, Guildford, Vol. 8, No. 4, pp.187-196.

Ferreira, P.M. and Liu, C.R.B,. 1986a, “An Analytical quadratic model for the geometric error of a machine tool". Journal of Manufacturing Systems, Dearborn, Vol. 5, No. 1, pp.51-62, Apr.

Ferreira, P.M. and Liu, C.R.B., 1986 ", "A Contribution to the analysis and compensation of the geometric error of a machining center". Annals of the CIRP, Paris, Vol. 35, No. 1, pp.259-262.

Kreng, V.B., Liu, C.R. and Chu, C.N., 1994, “A Kinematic model for machine tool accuracy characterization”. International Journal of Advanced Manufacturing Technology, London, No.9, pp.79-86.

Kruth, J.P., Vanherck, P. and Van Den Bergh, C., 2001, "Compensation of static and transient thermal errors on CMMs". Annals of the CIRP, Paris, Vol. 50, No.1, pp.377-380.

Pereira, P.H., 1995, "Levantamento e modelamento dos erros térmicos de uma retificadora cilíndrica CNC”. Dissertação (Mestrado) - Escola de Engenharia de São Carlos, Universidade de São Paulo, São Carlos. 156 p.

Ramesh, R., Mannan., M.A., and Poo, A.N., 2000, "Error compensation in machine tools - a review". Part II: Thermal errors. International Journal of Machine Tools \& Manufacture, Vol.40, pp.1257-1284.

Shivaswamy, S.C., 1992, "Error analysis of a three axis machine tool". UNC Charlotte.

Sosa Cardoza, J.A., 1995, "Máquinas Virtuais de Medir a Três Coordenadas”. Tese (Doutorado) - Escola de Engenharia de São Carlos, Universidade de São Paulo, São Carlos. 209 p.

Souza, V.A., 2000, "Calibração indireta de Máquinas de Medir a Três Coordenadas”. Dissertação (Mestrado) - Escola de Engenharia de São Carlos, Universidade de São Paulo, São Carlos, 141 p.

Valdés, A.R. 2003, "Modelo de sintetização de erros termicamente induzidos em Máquinas de Medir a Três Coordenadas”. Tese (Doutorado) Escola de Engenharia de São Carlos, Universidade de São Paulo, São Carlos, $191 \mathrm{p}$.

Vieira Sato, D.P. 1998, “Uma Contribuição ao modelo de sintetização de erros em máquinas ferramentas”. Tese (Doutorado) - Escola de Engenharia de São Carlos, Universidade de São Paulo, São Carlos, 198 p.

Wang Y.G., Zhang, K.S. and Moon, J.S., 1998, "Compensation for the thermal errors of a multi-axis machining center". ASME Transactions Journal of Materials Processing Technology, Vol.75, pp. 45-53.

Yuan, M. and Ni, J., 1998, "Measurement and prediction of thermal errors of a CNC machining center using two spherical balls". Journal of Materials Processing Technology. Vol. 75, Issue: 1-3, March 1, pp. 180-189. 